\title{
EUROfusion
}

EUROFUSION WPJET1-PR(15)29

J. Eriksson et al.

\section{Dual Sightline Measurements of MeV Range Deuterons with Neutron and Gamma-Ray Spectroscopy at JET}


"This document is intended for publication in the open literature. It is made available on the clear understanding that it may not be further circulated and extracts or references may not be published prior to publication of the original when applicable, or without the consent of the Publications Officer, EUROfusion Programme Management Unit, Culham Science Centre, Abingdon, Oxon, OX14 3DB, UK or e-mail Publications.Officer@euro-fusion.org".

"Enquiries about Copyright and reproduction should be addressed to the Publications Officer, EUROfusion Programme Management Unit, Culham Science Centre, Abingdon, Oxon, OX14 3DB, UK or e-mail Publications.Officer@euro-fusion.org".

The contents of this preprint and all other EUROfusion Preprints, Reports and Conference Papers are available to view online free at http://www.euro-fusionscipub.org. This site has full search facilities and e-mail alert options. In the JET specific papers the diagrams contained within the PDFs on this site are hyperlinked. 


\title{
Dual sightline measurements of $\mathrm{MeV}$ range deuterons with neutron and gamma-ray spectroscopy at JET
}

\author{
J. Eriksson ${ }^{1}$, M. Nocente ${ }^{2,3 *}$, F. Binda ${ }^{1}$, C. Cazzaniga $^{3,4}$, S. Conroy $^{1}$, G. Ericsson ${ }^{1}$, L.

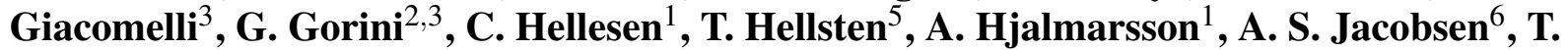 \\ Johnson $^{5}$, V. Kiptily ${ }^{7}$, T. Koskela ${ }^{8}$, M. Mantsinen ${ }^{9,10}$, M. Salewski ${ }^{6}$, M. Schneider ${ }^{11}$, S. \\ Sharapov $^{7}$, M. Skiba ${ }^{1}$, M. Tardocchi ${ }^{2,3}$, M. Weiszflog ${ }^{1}$ and JET Contributors ${ }^{\dagger}$ \\ EUROfusion Consortium, JET, Culham Science Centre, Abingdon, OX14 3DB, UK \\ ${ }^{1}$ Department of Physics and Astronomy, Uppsala University, Sweden \\ ${ }^{2}$ Dipartimento di Fisica 'G. Occhialini', Università degli Studi di Milano-Bicocca, Milano, Italy \\ ${ }^{3}$ Istituto di Fisica del Plasma 'Piero Caldirola', Associazione EURATOM-ENEA-CNR, Milano, Italy \\ ${ }^{4}$ ISIS Facility, Rutherford Appleton Laboratory, Science Didcot, UK \\ ${ }^{5}$ School of Electrical Engineering, KTH, Stockholm, Sweden \\ ${ }^{6}$ Technical University of Denmark, Department of Physics, DK-2800 Kgs. Lyngby, Denmark \\ ${ }^{7}$ CCFE, Culham Science Centre, Abingdon, UK \\ ${ }^{8}$ Department of Applied Physics, Aalto University, FI- 00076 AALTO, Finland \\ ${ }^{9}$ Catalan Institution for Research and Advanced Studies, Barcelona, Spain \\ ${ }^{10}$ Barcelona Supercomputer Center, Barcelona, Spain \\ ${ }^{11}$ CEA, IRFM, F-13108 Saint-Paul-lez-Durance, France
}

\begin{abstract}
Observations made in a JET experiment aimed at accelerating deuterons to the MeV range by third harmonic radiofrequency $(\mathrm{RF})$ heating coupled into a deuterium beam are reported. Measurements are based on a set of advanced neutron and gamma-ray spectrometers that, for the first time, observe the plasma simultaneously along vertical and oblique lines of sight. Parameters of the fast ion energy distribution, such as the high energy cut-off of the deuteron distribution function and the RF coupling constant, are determined from data within a uniform analysis framework for neutron and gamma-ray spectroscopy based on an one dimensional model and by a consistency check among the individual measurement techniques. A systematic difference is seen between the two lines of sight and is interpreted to originate from the sensitivity of the oblique detectors to the pitch-angle structure of the distribution around the resonance, which is not correctly portrayed within the adopted one dimensional model. A framework to calculate neutron and gamma-ray emission from a spatially resolved, two dimensional deuteron distribution specified by energy/pitch is thus developed and used for a first comparison with predictions from ab initio models of RF heating at multiple harmonics.

The results presented in this paper are of relevance for the development of advanced diagnostic techniques for MeV range ions in high performance fusion plasmas, with applications to the experimental validation of RF heating codes and, more generally, to studies of the energy distribution of ions in the MeV range in high performance deuterium and deuterium-tritium plasmas.
\end{abstract}

\section{Introduction}

One important aspect for the successful operation of a future burning fusion plasma, e.g. in ITER, is the ability to understand and control the behavior of fast ions in the plasma [1]. These ions, characterized by energies much

${ }^{*}$ Corresponding author. Email: massimo.nocente@mib.infn.it. The first two authors gave equal contributions to this work.

${ }^{\dagger}$ See the appendix of F. Romanelli et al., Proceedings of the 25th IAEA Fusion Energy Conference 2014, Saint Petersburg, Russia. higher than the average thermal energy, can excite and interact with a variety of plasma instabilities [2], which can affect the plasma performance and, in some cases, lead to energetic particle losses [3, 4].

The most important fast ion species at ITER will be the 3.5 MeV alpha particle produced in the $t(d, n)^{4} \mathrm{He}$ fusion reaction (DT). At JET, one opportunity for studying the behavior of fast ions in the $\mathrm{MeV}$ range is provided by combining neutral beam injection (NBI) with ion cyclotron resonance heating (ICRH) tuned to the 3rd harmonic of the 
cyclotron frequency of the NBI ion species. This technique has been used to accelerate both alpha particles [5] and deuterons $[6,7]$.

The fast ion energy distribution during 3rd harmonic ICRH can be studied by analysing the energy spectra of neutrons and gamma-rays emitted from the plasma. Neutrons and gamma-rays are produced in many reactions between the fast ions and other ion populations in the plasma, which means that the energy spectra of these reaction products depend on the velocity distribution of the reacting ions.

A 1-dimensional Fokker-Planck equation for the fast ion distribution, adapted from reference [8], has previously been successfully used to interpret neutron and gamma-ray measurements of JET plasmas heated with 3rd harmonic ICRH. In reference [9] this equation was solved within the framework of cold plasma theory, using input from experimentally measured quantities (the most important parameters being the electron density, the coupled ICRH power and the NBI power). It was found that fast deuteron energy distributions calculated in this way were in good agreement with distributions inferred from measurements of the neutron spectrum from the $d(d, n)^{3} \mathrm{He}$ reaction (DD). In reference [10] the same framework was used to calculate the distribution functions of deuterons and alpha particles during a JET experiment with ${ }^{4} \mathrm{He}$ NBI. The calculations were consistent with the measured spectrum of neutrons from the DD and ${ }^{9} \mathrm{Be}(\alpha, \mathrm{n} \gamma)^{12} \mathrm{C}$ reactions. In both these past experiments, only measurements from a single detector observing the plasma from a vertical line of sight were available.

The above mentioned Fokker-Planck modeling depends on the perpendicular wave number of the ICRH wave field $\left(k_{\perp}\right)$ as well as on the strength of the quasi-linear diffusion coefficient representing wave-particle interaction $\left(C_{\mathrm{RF}}\right)$, as described in section 3.1. In reference [11] the value of $k_{\perp}$ could be estimated from measurements of the gamma-ray peaks from the ${ }^{9} \mathrm{Be}(\alpha, \mathrm{n} \gamma){ }^{12} \mathrm{C}$ and ${ }^{12} \mathrm{C}(\mathrm{d}, \mathrm{p} \gamma){ }^{13} \mathrm{C}$ reactions. The same reactions were studied in [12], but in this case $C_{\mathrm{RF}}$ rather than $k_{\perp}$ was estimated from the data.

Motivated by the previous results, the aim of the work presented in this paper is to develop a framework for performing a uniform analysis of fast ion measurements with neutron (NES) and gamma-ray spectroscopy (GRS). For the first time, we combine measurements from several neutron and gamma-ray spectrometers observing the plasma along two different lines of sight - one vertical and one oblique - with the aim of studying the additional capabilities to diagnose the fast ion energy distribution that are offered by such combination. The measurements in the vertical line of sight are performed with the time of flight neutron spectrometer TOFOR [13] and the High Purity Germanium $(\mathrm{HpGe})$ high resolution gamma-ray spectrometer $[14,15]$. In the oblique line of sight, a NE213 liquid scintillator [16] and a diamond detector [17, 18] are used to measure the neutron spectrum. The analysis is carried out by starting from a modeled fast ion distribution and calculating the expected spectra seen by the different instru- ments, taking the different sightlines and instrumental response functions into account. This allows a consistent comparison of the data from the different diagnostics, by investigating if a given fast ion distribution is compatible with the measurements. The measurements analysed in the paper was collected during JET experiments with 3rd harmonic ICRH and deuterium NBI, carried out in the summer of 2014. In particular, we find that the combination of the two lines of sight makes our measurements sensitive to the two dimensional energy-pitch structure of the energy distribution in the $\mathrm{MeV}$ range. This opens up to the use of our data for a validation of ICRH models of RF heating at multiple harmonics in the $\mathrm{MeV}$ range with an unprecedented detailed level, which is of relevance for accurately testing the heating schemes expected in ITER, as well as, more generally, for detailed studies of the energy distribution of fusion products and energetic ions in the $\mathrm{MeV}$ range. JET and the set of nuclear diagnostics described here are unique in this respect, as no other machine is presently capable to generate, confine and study in detail the distribution of energetic ions in the same range energy range expected for $\alpha$ particles born from fusion reactions in a deuterium-tritium plasma.

The paper is organized as follows. A description of the JET discharges under consideration as well as the relevant neutron and gamma-ray diagnostics is given in section 2 . The model of the fast deuteron distribution and the data analysis procedure are described in section 3 . The results are presented in section 4 and discussed in section 5 with special emphasis on the pitch angle sensitivity offered by the the two lines of sight. A framework for comparison with ab initio models of ICRH in plasmas is finally developed and a first comparison with theoretical predictions is shown. The conclusions of the paper are presented in section 6.

\section{Experimental setup}

\subsection{JET discharges}

Three JET discharges $(86459,86461$ and 86464) are studied in this paper. The magnetic field on-axis was $2.25 \mathrm{~T}$ for all discharges and the frequency of the ICRH was 51 $\mathrm{MHz}$. With these settings the location of the 3rd harmonic deuterium cyclotron resonance is in the plasma center, at a major radius between 3 and 3.1 meters. Discharges 86459 and 86461 were similar to each other, both having a central electron density of about $4 \cdot 10^{19} \mathrm{~m}^{-3}$ and subject to 3 MW of ICRH and 4.5 MW of NBI. Discharge 86464 had a higher electron density, about $5 \cdot 10^{19} \mathrm{~m}^{-3}$, and was heated with $4 \mathrm{MW}$ of ICRH and $2.5 \mathrm{MW}$ of NBI.

Time traces of the measured neutron rates, heating powers and electron densities for the three discharges are shown in figure 1 . It can be noted that although the ICRH and the NBI powers are similar in magnitude, the neutron rate increases about 5-10 times when the ICRH is applied. This is because the ICRH couples to the beam ions and ac- 

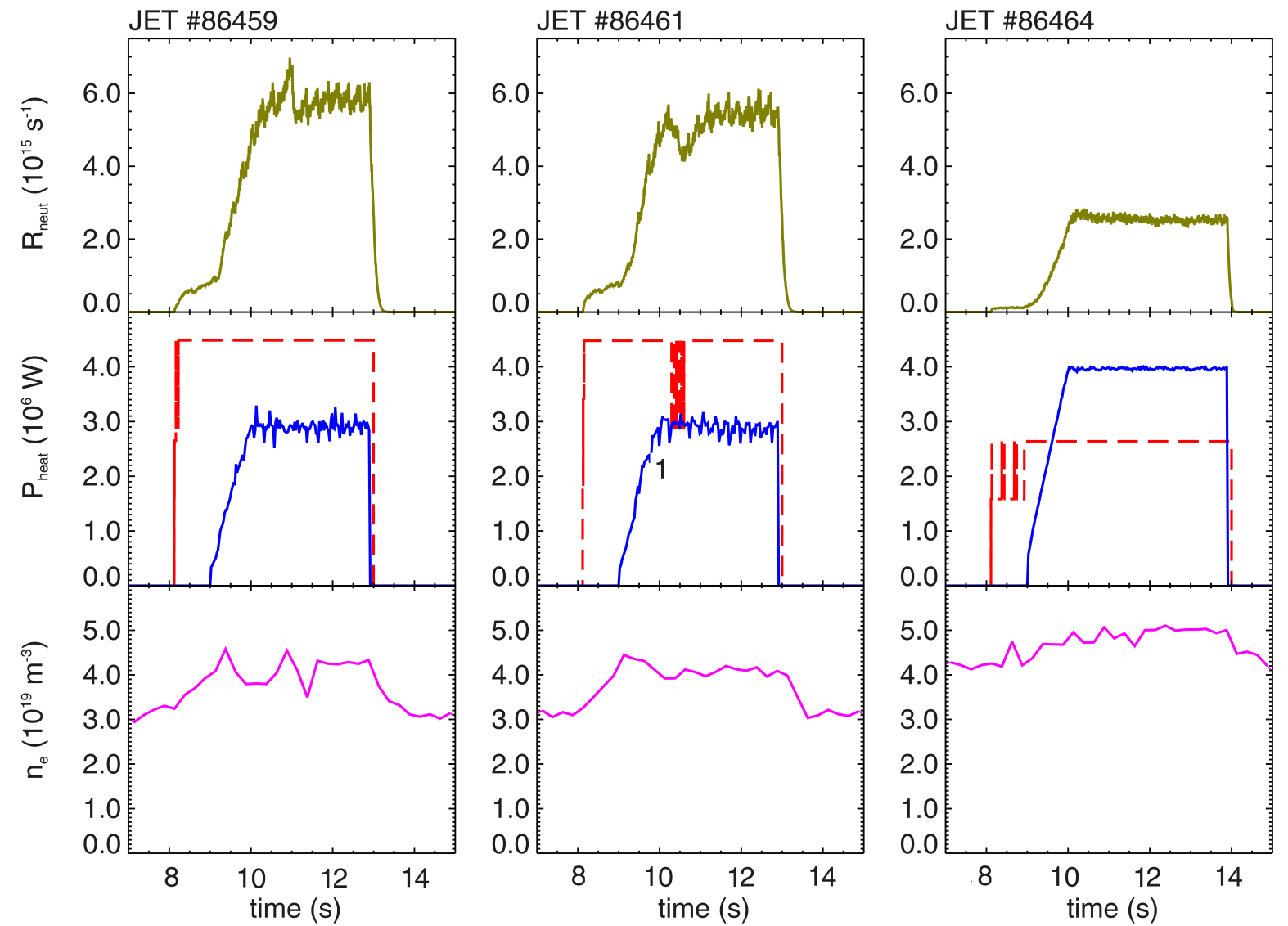

Figure 1: Overview of the JET discharges analyzed in this paper. The top panel shows the total neutron rate as measured by the fission chambers, the middle panel shows the applied heating power from NBI (dashed line) and ICRH (solid line) and the bottom panel shows the central electron density. The time window is limited to the heating phase.

celerates a significant number of ions to kinetic energies in the $\mathrm{MeV}$ range, where the DD fusion cross section is about 5 times larger than at the NBI energy $(\sim 100 \mathrm{keV})$. This strong synergy between 3rd harmonic ICRH and NBI is well known from previous experiments [6,9]. The dominating contribution to the neutron emission is therefore due to beam-target reactions involving fast deuterons and ions from the background plasma.

\subsection{Neutron spectrometers}

Three neutron spectrometers were used in the experiment. One of the neutron spectrometers is the time-of-flight spectrometer TOFOR, described in detail in reference [13]. TOFOR is situated in the roof laboratory, 19 meters above the JET tokamak, and was installed in 2005. The sightline is perpendicular to the magnetic field. TOFOR consists of two sets of plastic scintillator detectors, S1 and S2. The $\mathrm{S} 1$ detectors are located in the collimated neutron beam whereas S2 detectors are placed 1.2 meters away from S1 at an angle of 30 degrees to the beam direction. Some of the incoming neutrons will scatter in the $\mathrm{S} 1$ detector. If the scattering angle of a given neutron is about 30 degrees it is possible that this neutron scatters also in the $\mathrm{S} 2$ detector.
The time of each scattering event is recorded and from the the two arrays of scattering times a time-of-flight spectrum is constructed.

The time-of-flight of a neutron is related to its energy. DD neutrons, which typically have energies of about 2.5 $\mathrm{MeV}$, give rise to flight times around $65 \mathrm{~ns}$. The full response function of TOFOR has been calculated with Monte-Carlo methods. Detailed knowledge of the response function is crucial for the analysis presented in this paper, in order to calculate the expected measured spectrum corresponding to different fast ion distributions.

Two additional, compact spectrometers were available on an oblique line of sight. The first of these two instruments is an NE213 organic liquid scintillator, which has been in operation at JET since 2012 [16]. The NE213 is located in the back of the upgraded Magnetic Proton Recoil (MPRu) spectrometer [19] and has an oblique sightline that makes a double pass through the plasma core. The angle between the magnetic field and the sightline is 47 degrees at the magnetic axis. The NE213 scintillator is connected to a photomultiplier (PM) tube and a digital data acquisition system. A neutron that scatters in the detector gives rise to scintillation light which results in a voltage pulse 
from the PM tube. The full wave form of the pulse from the PM tube is digitized and recorded. The pulse height is related to the energy deposited by the neutron in the detector and the pulse height spectrum is therefore related to the energy spectrum of the neutrons incident on the detector. The response function of this system has been modeled with Monte-Carlo calculations and is further calibrated with a ${ }^{22} \mathrm{Na}$ gamma-ray source and neutrons from plasmas heated exclusively by ohmic heating, as described in [16].

Placed in front of the NE213 and thus sharing the same line of sight, a synthetic chemical vapour deposition diamond detector (CVD) was also used for neutron measurements [17, 18]. Similarly to the NE213, the neutron spectrum is measured via the energy that is deposited in the device when incoming neutrons scatter elastically on ${ }^{12} \mathrm{C}$ nuclei of the CVD. A set of two fast pre-amplifiers and a digitizer [17] are used to convert each pulse from $n+{ }^{12} \mathrm{C}$ interactions into a digitized waveform that is then integrated to reconstruct the spectrum of the energy deposited in the device after each discharge. The instrument response function is known accurately from MCNP simulations and has been benchmarked against NBI plasmas [17]. A triple alpha source $\left({ }^{241} \mathrm{Am},{ }^{239} \mathrm{Pu}\right.$ and $\left.{ }^{244} \mathrm{Cm}\right)$ is used for energy calibration before and during each plasma discharge.

\subsection{High resolution gamma-ray spectrometers}

The set of high resolution gamma-ray spectrometers consists of High Purity Germanium (HpGe) [14, 15] and $\mathrm{LaBr}_{3}(\mathrm{Ce})$ detectors $[20,21,22,23]$. Both spectrometers are situated in the roof lab with the same line-of-sight as TOFOR and can be alternatively selected for use before each discharge. HpGe features the best instrumental resolution (about $1 \mathrm{keV}$ for gamma-ray energies of a few $\mathrm{MeV}$ ), allowing one to distinguish the spectral broadening of characteristic peaks from nuclear reactions between fast ions and impurities [14, 15, 24], which can be used to extract information on the fast ion energy distribution. $\mathrm{LaBr}_{3}$ has comparably a coarser resolution (about 20-40 keV at the same energies), but it is about two times more efficient and can sustain counting rates up to few $\mathrm{MHz}$ [21], of relevance for $\alpha$ particle studies in deuterium-tritium plasmas. $\mathrm{MHz}$ counting rates cannot be coped with by HpGe.

Both detectors were used in the experiment, where the reactions of interest are those between the fast deuterons and ${ }^{9} \mathrm{Be}$ impurities naturally found in the plasma due to the ITER like wall [25]. The resulting emission spectrum is quite rich, as it is made of several peaks from transitions between excited states of ${ }^{10} \mathrm{~B}$ and ${ }^{10} \mathrm{Be}$ generated in the ${ }^{9} \mathrm{Be}(\mathrm{d}, \mathrm{n} \gamma){ }^{10} \mathrm{~B}$ and ${ }^{9} \mathrm{Be}(\mathrm{d}, \mathrm{p} \gamma){ }^{10} \mathrm{Be}$ reactions, respectively. An example of the sum spectrum measured with the HpGe detector in the discharges considered in this paper is shown in figure 2. In the following, we will focus on the $2868 \mathrm{keV}$ and $3367 \mathrm{keV}$ lines, that correspond to the transitions from the 4 th to 1 st state of ${ }^{10} \mathrm{~B}$ and 1 st to ground state of ${ }^{10} \mathrm{Be}$, respectively (see figure 3 ). The choice is motivated by the enhanced intensity offered by these two transitions compared to other transitions from the same nuclei and a rather good availability of cross section data, retrieved from the EXFOR database*. The data is necessary for the interpretation of gamma-ray Doppler broadening and also includes information on cross section anisotropies, which play a crucial role in determining the spectral shape of characteristic gamma-ray emission peaks from nuclear reactions $[26,27]$.

\section{Method}

\subsection{Modeling the fast ion distribution}

The velocity distribution $f(\mathbf{v}, t)$ of ICRH accelerated ions in a Maxwellian background plasma can be described by a Fokker-Planck equation of the form

$$
\frac{\partial f}{\partial t}=C(f)+Q(f)+S(\mathbf{v})+L(\mathbf{v}) .
$$

Here, $C(f)$ represents the effect of collisions between the fast ions and the background plasma particles. $Q(f)$ represents the interaction between the fast ions and the wave field produced by the ICRH, which can be described as a quasilinear diffusion process [28]. The terms $S(\mathbf{v})$ and $L(\mathbf{v})$ are included to account for sources and losses of fast ions, respectively.

Since the gyrophase is an ignorable coordinate, the velocity of an ion in the plasma can be decomposed into the components parallel $\left(v_{\|}\right)$and perpendicular $\left(v_{\perp}\right)$ to the background magnetic field. The ICRH scheme affects mainly the perpendicular component of the ion velocity. Hence, for an ion accelerated to energies much higher than the thermal energy, the fast ion velocity distribution will be strongly anisotropic, with $v_{\perp} \gg v_{\|}$. In this limit it is possible to write the Fokker-Planck equation (1) as a one dimensional equation for $f\left(v_{\perp}, t\right)[8,29]$,

$$
\begin{aligned}
& \frac{\partial f}{\partial t}=\frac{1}{v_{\perp}} \frac{\partial}{\partial v_{\perp}}\left[-\alpha v_{\perp} f+\frac{1}{2} \frac{\partial}{\partial v_{\perp}}\left(\beta v_{\perp} f\right)+\frac{1}{4} \gamma f+\right. \\
& \left.D_{\mathrm{RF}} v_{\perp} \frac{\partial f}{\partial v_{\perp}}\right]+S\left(v_{\perp}\right)+L\left(v_{\perp}\right) \text {. }
\end{aligned}
$$

In the above equation, $\alpha, \beta$ and $\gamma$ are the Coulomb diffusion coefficients as given by Spitzer [30] and $D_{\mathrm{RF}}$ is the quasilinear radio frequency (RF) diffusion coefficient. The latter is given by

$$
D_{\mathrm{RF}}=C_{\mathrm{RF}}\left|J_{n-1}\left(\frac{k_{\perp} v_{\perp}}{\omega_{\mathrm{ci}}}\right)+\frac{E_{-}}{E_{+}} J_{n+1}\left(\frac{k_{\perp} v_{\perp}}{\omega_{\mathrm{ci}}}\right)\right|^{2}
$$

where $k_{\perp}$ is the perpendicular wave number of the RF wave field and $\omega_{\mathrm{ci}}$ is the cyclotron frequency of the resonating ions. $C_{\mathrm{RF}}$ is a constant proportional to the absorbed RF power per particle and $E_{ \pm}$denote the left handed (+) and right handed (-) component of the electric field at the resonance. $J$ denotes Bessel functions of the first kind, for which the index $n=1,2,3 \ldots$ is the harmonic number of the

\footnotetext{
*https://www-nds.iaea.org/exfor/exfor.htm
} 


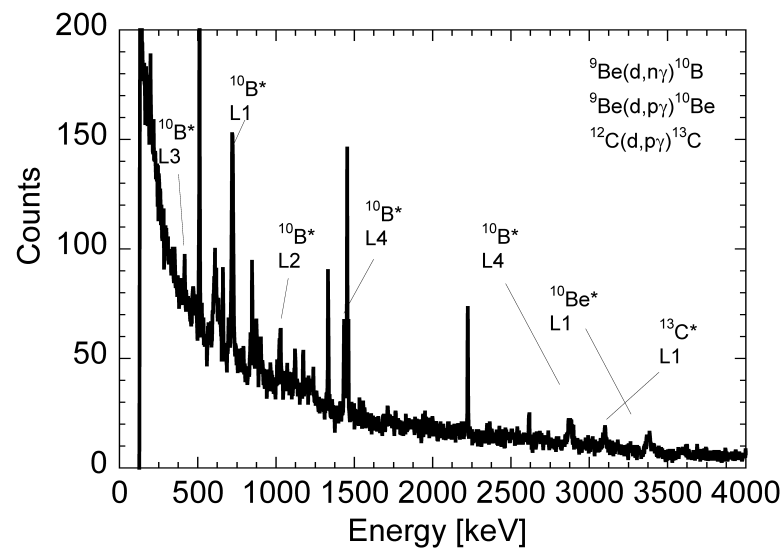

Figure 2: Sum spectrum measured with the High purity Germanium detector in the discharges analyzed in this paper. The main peaks resulting from different reactions between deuterons and ${ }^{9} \mathrm{Be}$ impurities are indicated by the nuclei responsible for the emission as well as their excited states. A peak from the ${ }^{12} \mathrm{C}(\mathrm{d}, \mathrm{p} \gamma){ }^{13} \mathrm{C}$ reaction at $E_{\gamma}=3089 \mathrm{keV}$ was also observed and is due to the presence of residual ${ }^{12} \mathrm{C}$ impurities in the plasma.
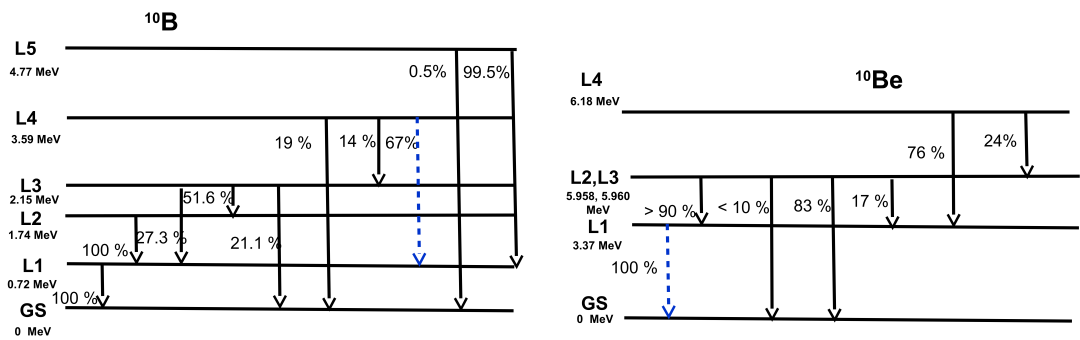

Figure 3: Energy levels of the nuclei ${ }^{10} \mathrm{~B}$ and ${ }^{10} \mathrm{Be}$ produced in the ${ }^{9} \mathrm{Be}(\mathrm{d}, \mathrm{n} \gamma){ }^{10} \mathrm{~B}$ and ${ }^{9} \mathrm{Be}(\mathrm{d}, \mathrm{p} \gamma){ }^{10} \mathrm{Be}$ reactions, respectively, with their branching ratios indicated. The transitions from 4 th to 1 st state of ${ }^{10} \mathrm{~B}$ and from 1 st to ground state of ${ }^{10} \mathrm{Be}$, leading to gamma-ray peaks at $2868 \mathrm{keV}$ and $3367 \mathrm{keV}$, respectively, are marked by dashed blue lines.

heating and $n=3$ for the plasma scenario studied in this paper.

The Spitzer coefficients can be calculated from measurements of properties of the background plasma, such as the ion and electron temperatures $T_{\mathrm{i}, \mathrm{e}}$, the electron density $n_{\mathrm{e}}$ and the effective charge $Z_{\text {eff }}$. The electric field polarization $E_{-} / E_{+}$is here calculated in the cold plasma approximation, using the relations given in [31].

Under stationary conditions $(\partial f / \partial t=0)$, equation (2) reduces to a first order ordinary differential equation, which is readily solved analytically by standard techniques. The solution is a function of $v_{\perp}$ but can also be represented as a function of the ion energy $E$, given by $m v_{\perp}^{2} / 2$ (since $\left.v_{\perp} \gg v_{\|}\right)$. With $\alpha, \beta, \gamma$ and $E_{-} / E_{+}$calculated as described above, the solution $f\left(v_{\perp}\right)$ depends only on the values of $k_{\perp}$ and $C_{\mathrm{RF}}$. These two parameters affect two prominent features of the distribution, as described in what follows.

The value of $k_{\perp}$ determines the location of the first zero of the diffusion coefficient $D_{\mathrm{RF}}$, as seen from equation (3). The energy at which this zero occurs is denoted by $E^{*}$ in this paper. Very few ions can be accelerated above this energy and hence this results in a cut-off in the distribution $[32,29]$, as exemplified in the bottom panel of figure 4, which shows calculated fast deuteron distributions for sev- eral values of $k_{\perp}$. It is clearly seen that the $E^{*}$ changes as $k_{\perp}$ is varied.

As stated in the beginning of this section, $C_{\mathrm{RF}}$ is proportional to the absorbed power per particle. Hence, a higher value of $C_{\mathrm{RF}}$ will give a higher probability for an ion to be accelerated to high energies, i.e. the tail of the distribution will be more pronounced. This is illustrated in the top panel of figure 4.

\subsection{Calculation of neutron and gamma-ray spectra}

Starting from the model for the fast ion distribution described above it is possible to calculate the shapes of the neutron and gamma-ray energy spectra seen by the instruments described in section 2.2. This is done by means of Monte-Carlo simulations [33, 27] sampling the fast deuteron distribution and the background plasma ions (deuterons and beryllium, for the neutron and gamma-ray spectra, respectively) and calculating the corresponding reaction product energy. For the 3rd harmonic ICRH scenario under consideration, it is also necessary to take finite Larmor radii (FLR) effects into account when performing calculations for the vertical sightlines of TOFOR and the HpGe spectrometer, as shown in $[34,11]$. This is due to 


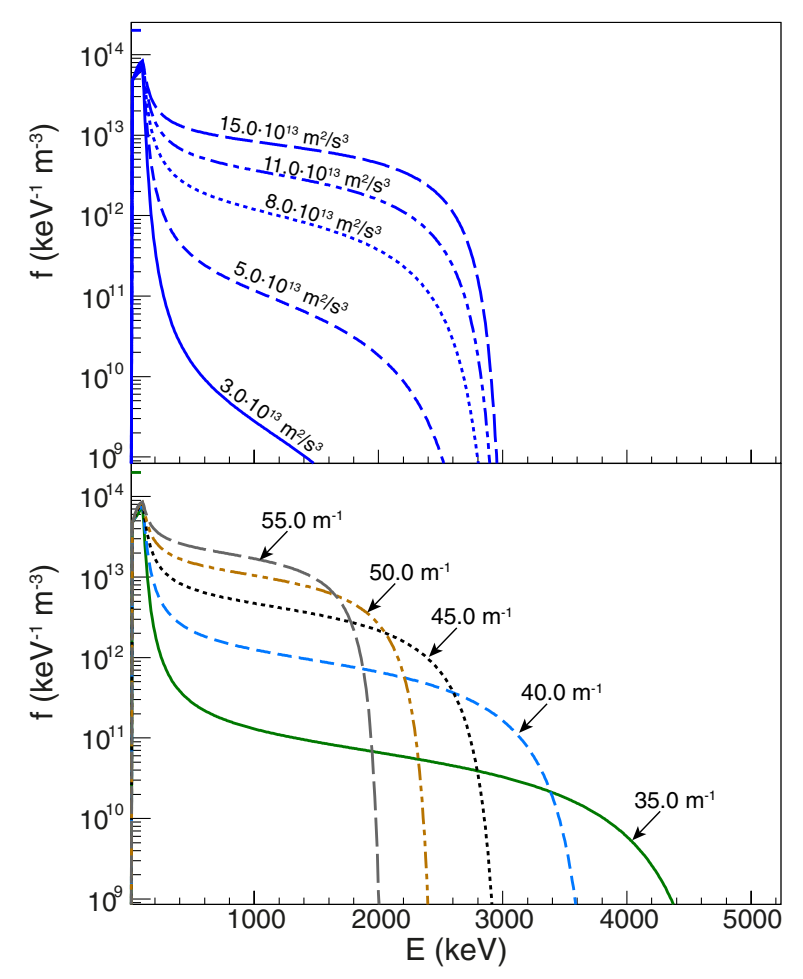

Figure 4: Fast deuteron energy distribution during 3rd harmonic ICRH and NBI, calculated from equation (2). Top: $k_{\perp}=45.0$ $\mathrm{m}^{-1}$ and $C_{\mathrm{RF}}$ ranging from $3 \cdot 10^{13} \mathrm{~m}^{2} / \mathrm{s}^{3}$ to $15 \cdot 10^{13} \mathrm{~m}^{2} / \mathrm{s}^{3}$. Bottom: $k_{\perp}$ ranging from $35 \mathrm{~m}^{-1}$ to $55 \mathrm{~m}^{-1}$ and $C_{\mathrm{RF}}=12 \cdot 10^{13}$ $\mathrm{m}^{2} / \mathrm{s}^{3}$.

the high reactant energies involved and the large Larmor radius of the poloidal projection of the particle orbit compared to the width of the TOFOR and HpGe line of sight. For the NE213 and CVD spectrometers, these FLR effects are not important, as their sight line is oblique and broader than the projected fast ion Larmor radius.

Apart from the energy distribution obtained by solving equation (2), the spectra are also sensitive to the pitch angle distribution of the fast ions. As remarked in the beginning of section 3.1, the fast ion distribution obtained within the 1 dimensional model is strongly anisotropic in velocity space, due to the assumption $v_{\perp} \gg v_{\|}$. This corresponds to pitch angles close to 90 degrees. In previous neutron and gamma spectrometry analysis of similar plasma scenarios, pitch angles distributed as a Gaussian function with FWHM between 80 and 100 degrees were assumed in the calculations $[35,36,9]$ to account for the pitch angle anisotropy and this is assumed also in the present paper. The fit sensitivity to specific assumptions on the pitch angle is discussed later on in section 5 . The absolute value of the background plasma density is not of importance for the spectrum calculation, since this only affects the overall normalization, which is left as a free parameter in the fit when comparing with measurements. This is in contrast to the calculation of the Spitzer coefficients and the electric field polarization, which do depend on the details of the bulk plasma. The spectra calculated in this way depend directly on the $k_{\perp}$ and $C_{\mathrm{RF}}$ used for the fast ion distribution, that are determined by the values that give the best fit to data.

\subsection{Data analysis procedure}

The calculated neutron and gamma-ray spectrum histograms are multiplied with the instrumental response functions and compared with the measured data. For the TOFOR, NE213 and diamond spectrometers, the model spectra are fitted to the data, using $k_{\perp}, C_{\mathrm{RF}}$ and the overall normalization as free parameters. A component describing scattered neutrons is also included in the analysis of TOFOR [37]. In addition to the best fit values of the parameters, unconstrained statistical uncertainties for each parameter are also obtained from the fitting procedure, by a Monte-Carlo mapping of the likelihood function around the optimum.

A similar analysis is carried out for the HpGe gammaray spectrometer. However, for this instrument the fitted results are less significant, due to quite limited statistics for the discharges under consideration. Therefore, no attempt was made to adjust $k_{\perp}$ and $C_{\mathrm{RF}}$ to find the best fit for this instrument; these values were simply taken from the TOFOR analysis and kept fixed during the fitting procedure (leaving only the overall intensity as the free parameter). The fits obtained in this way give an indication of whether the distribution function estimated from the TOFOR data is also consistent with the HpGe results.

The above procedure was carried out for the three JET discharges described in section 2 . The results are presented and discussed in the following sections.

\section{Results}

The data and the fitted spectral components for the two instruments, from which the $C_{R F}$ and $k_{\perp}$ parameters of the 1 dimensional model were derived, are shown in figures 5 and 6, respectively. In figure 5 we show the time of flight spectra recorded with TOFOR to the left and the fitted neutron energy spectra to the right for the three discharges. Short times of flight correspond to large neutron energies. In the two low density discharges we record times of flight down to $40 \mathrm{~ns}$ corresponding to neutron energies up to 5.7 $\mathrm{MeV}$. However, in the high density discharge the times of flight at significant counting rates were observed to $44 \mathrm{~ns}$ corresponding to neutron energies up to $5.2 \mathrm{MeV}$. Figure 6 presents the measurement and inferred neutron energies for the diamond detector. Also here we observe significant count rates in the outer channels for the low density discharge but not for the high density discharge. The corresponding maximum neutron energies were $5.2 \mathrm{MeV}$ and 4.8 MeV, respectively.

The inferred values of the $C_{R F}$ and $k_{\perp}$ parameters (with their uncertainties) were used to calculate the corresponding deuteron energy distributions from equation 2 and are 

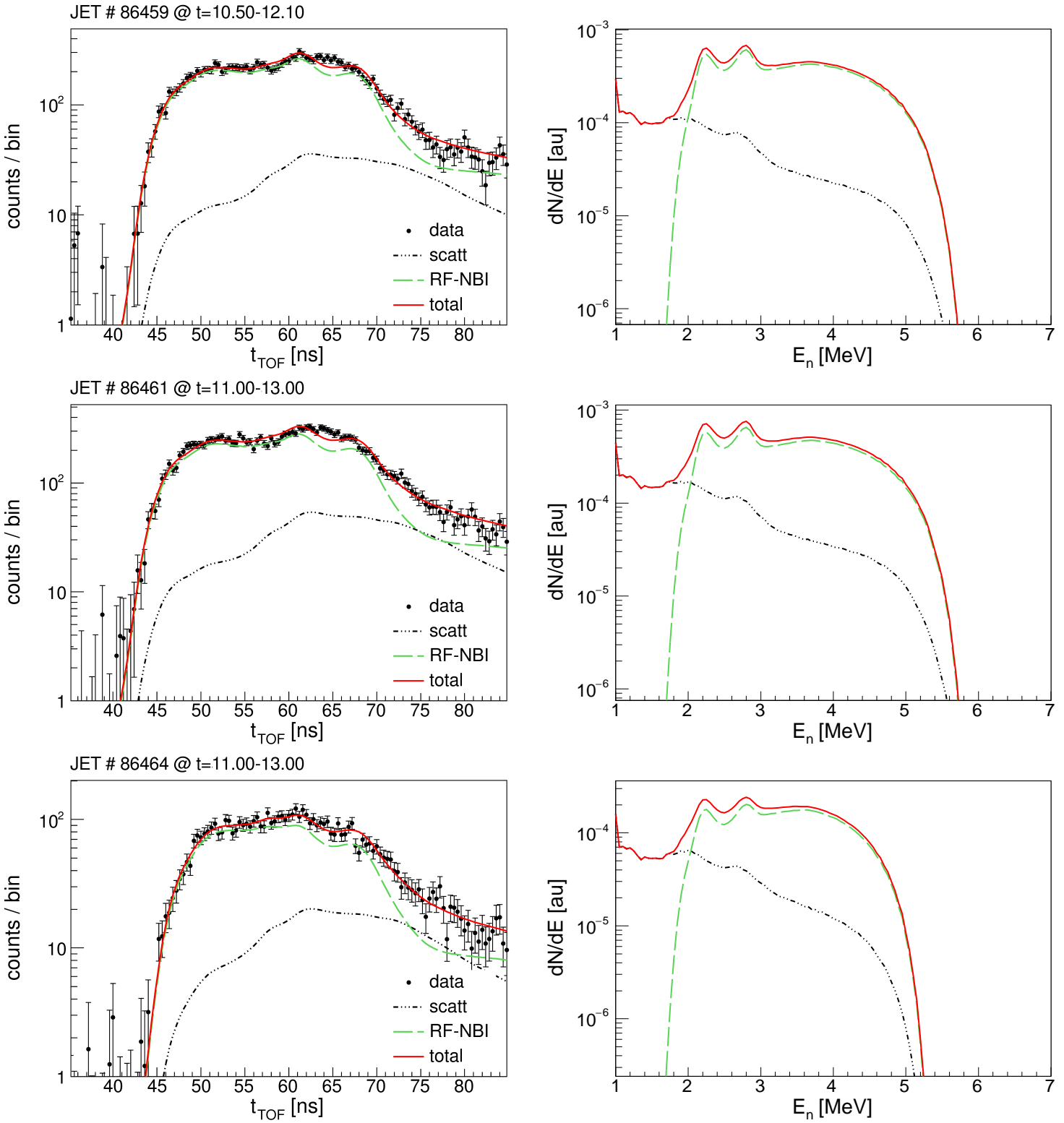

Figure 5: TOFOR data for JET discharges 86459,86461 and 86464 . Neutron spectra calculated from the modelling described in section 3 has been fitted to the data, along with a component describing scattered neutrons. The left panel shows the data and the fitted components and the right panel shows the components on neutron energy scale. The corresponding fast deuteron distributions are shown in figure 7. Measurements were performed along a vertical line of sight (see text for details). 

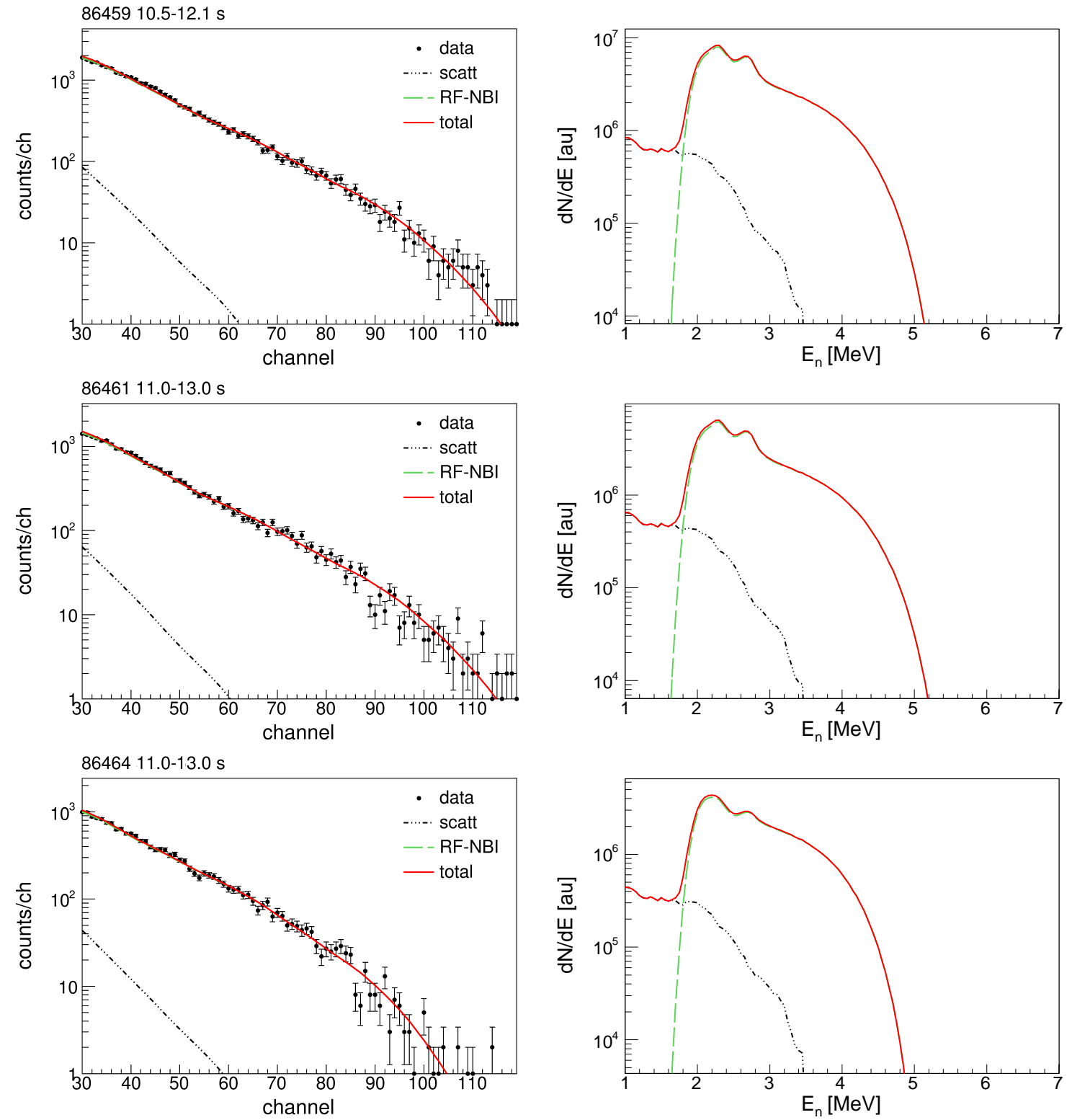

Figure 6: NE213 data for JET discharges 86459,86461 and 86464 . Neutron spectra calculated from the modeling described in section 3 have been fitted to the data, along with a component describing scattered neutrons. The left panel shows the data and the fitted components and the right panel shows the components on neutron energy scale. The corresponding fast deuteron distributions are shown in figure 7. Measurements were performed along an oblique line of sight (see text for details). 

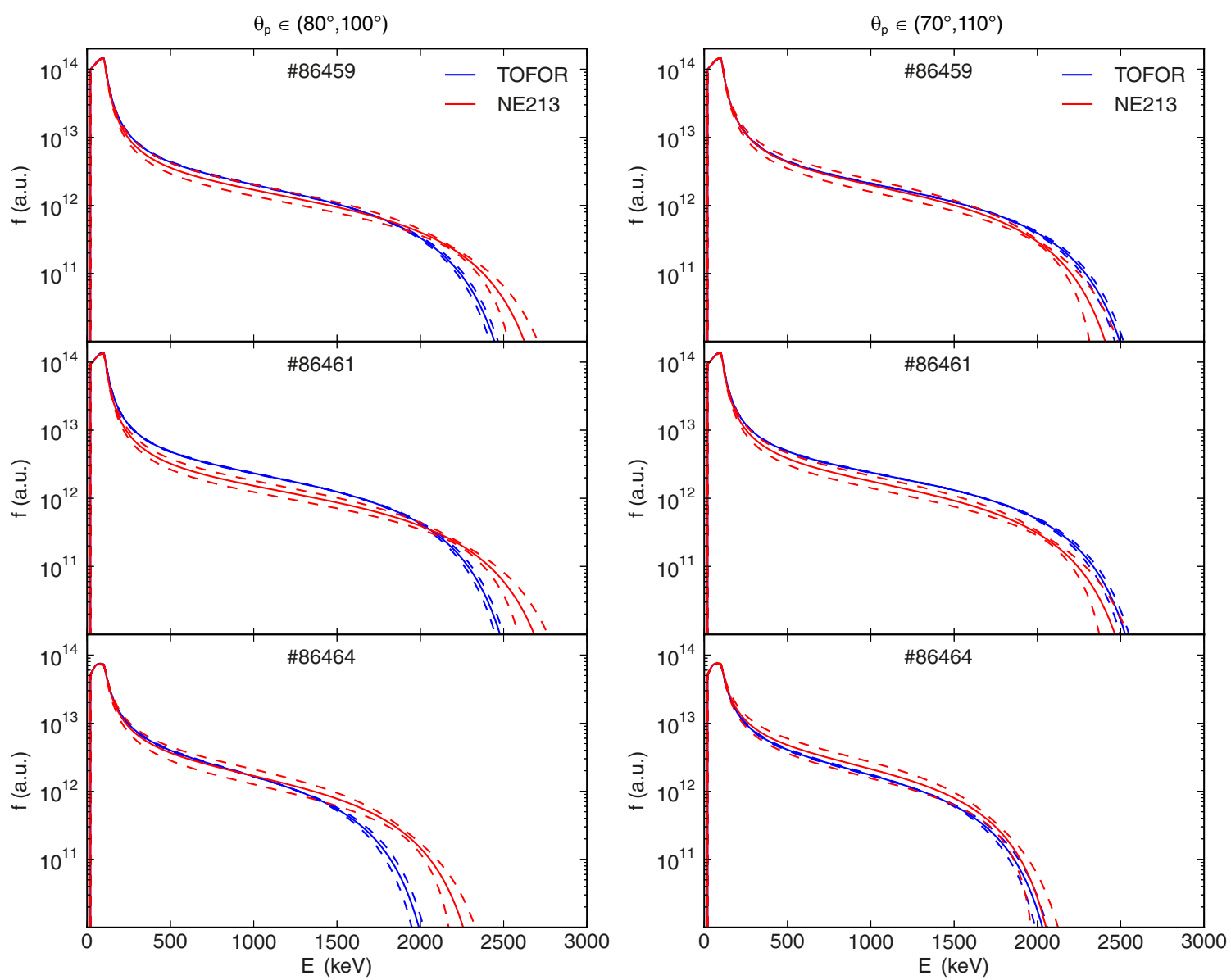

Figure 7: Fast deuterium distributions obtained from the TOFOR (vertical line of sight, blue) and NE213 (oblique line of sight, red) fits. The dashed lines are the estimated uncertainty as explained in the text. The left panel is the result when assuming pitch angles $\theta_{\mathrm{p}}$ in the range $80-100$ degrees and the right panel is for $70-110$ degrees (see discussion). The measured data and the fits are shown in figures 5 and 6 .

shown in figure 7. The solid lines represent the best-fit distributions and the dashed lines indicate the statistical uncertainty from the fit. For the NE213, the dashed lines also include uncertainties introduced by limited knowledge of the proton light output function for the scintillator, which is needed in order to construct the NE213 response function [38]. Additional systematic uncertainties, introduced by the limitations in the modelling of the distribution function, are further discussed in section 5.

A comparison of the distributions derived from data of different discharges reveals that similar fast deuteron populations were present in discharges 86459 and 86461, which had an energy cut-off $E^{*}$ around $2.5 \mathrm{MeV}$ based on the TOFOR fits. The fast deuteron distribution of discharge 86464 was instead different and had an $E^{*}$ about $500 \mathrm{keV}$ lower, which may be mostly due to the higher electron density obtained in this case. Similarly to the former experiments $[7,9,11]$, the measured cut-off energies clearly demonstrate the acceleration of deuterons to the $\mathrm{MeV}$ energy range.

By comparing the $C_{R F}$ and $k_{\perp}$ parameters derived from the TOFOR and NE213 analysis (figure 8), we however find that values derived from the TOFOR analysis are systematically lower than the corresponding ones from the NE213, and the difference is well beyond the error bars derived from fitting. In order to understand whether this discrepancy was due to inconsistencies in the detector settings, we have verified whether the same distribution that fits the TOFOR data could be used to describe data of the HpGe. Since the TOFOR and the HpGe have a common line of sight, they observe the same plasma volume and must therefore yield consistent results. A gamma-ray spectrum from the $\mathrm{HpGe}$ spectrometer with sufficient statistics was available only for discharge 86461 . Thus, for this discharge, the deuteron distribution estimated from the TOFOR analysis was used to calculate the expected gamma spectrum with results shown in figure 9.

The calculated spectral shapes for the gamma-ray peaks at $2868 \mathrm{keV}$ and $3367 \mathrm{keV}$ are in very good agreement with the measured data, with a normalised chi square of about 0.8. The simulations are also capable to reproduce the measured ratio $r$ of counts in the 2868 and $3367 \mathrm{keV}$ peaks, as they predict $r=0.91$, that compares well with a measured $r=0.7 \pm 0.2$ for 86461 and $r=0.85 \pm 0.08$ for 86459 . In 

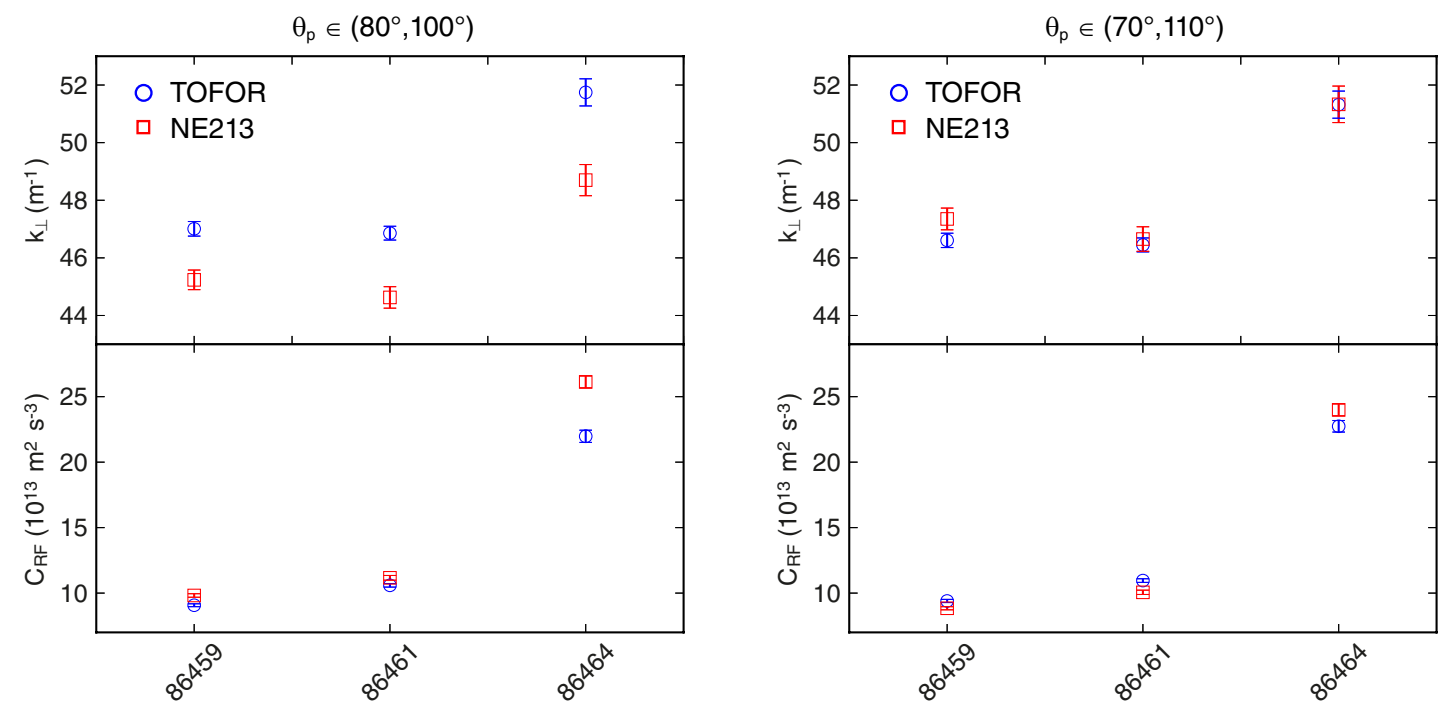

Figure 8: Fitted values of $k_{\perp}$ and $C_{\mathrm{RF}}$ from the TOFOR (blue circles) and NE213 (red squares) analysis. The left panel is the result when assuming pitch angles $\theta_{\mathrm{p}}$ in the range $80-100$ degrees and the right panel is for $70-110$ degrees.
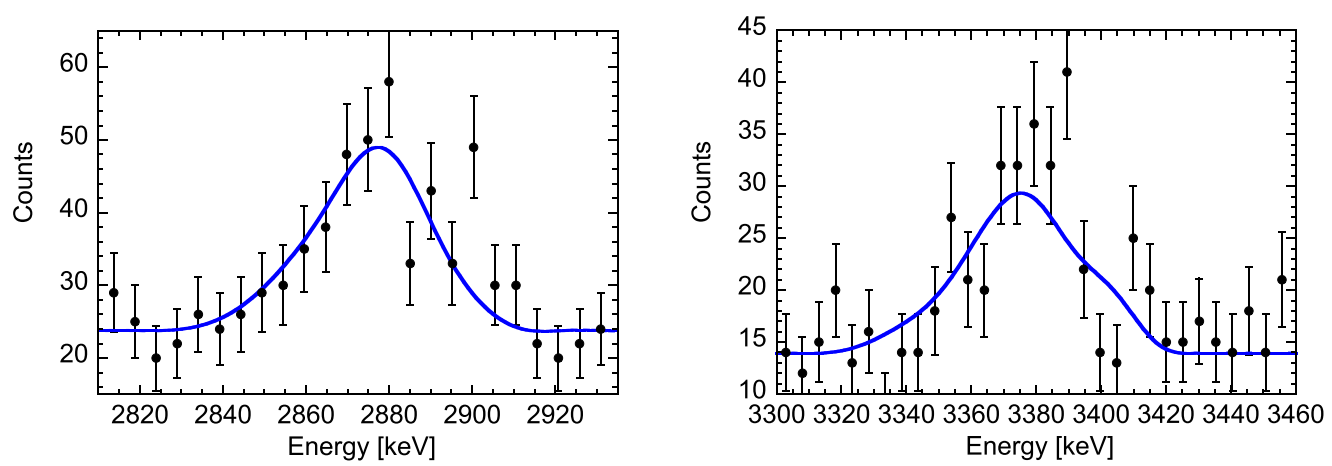

Figure 9: HpGe measurements of the $2868 \mathrm{keV}$ (left) and the $3367 \mathrm{keV}$ (right) gamma-ray peaks in JET discharge 86461 . A calculated gamma-ray spectrum, using the deuteron distribution obtained from the TOFOR analysis, have been fitted to the data (blue line).

the latter discharge, instead of the $\mathrm{HpGe}$, the $\mathrm{LaBr}_{3}$ detector was used, the higher efficiency of which is reflected in a smaller error bar for the peak ratio. On the other hand, with $\mathrm{LaBr}_{3}$ we could not measure details of the peak shape, as the instrumental energy resolution of the detector at the energies of interest for the analysis (between 1.6 and $1.7 \%$ ) was comparable with the expected peak Doppler broadening (about $1.3 \%$ full width at half maximum) from the reactant kinematics. The overall, experimental peak widths measured with $\mathrm{LaBr}_{3}$ in 86459 were $2.2 \pm 0.2 \%$ and $1.9 \pm 0.1 \%$ for the 2868 and $3367 \mathrm{keV}$ peaks, respectively, which are compatible with the peak broadening expected by combination of the instrumental and kinematics contributions.

A similar consistency analysis was performed by comparing results of the NE213 and CVD detectors, as they also measure neutron emission along the same line of sight. A full description of the CVD measurements, their analysis and sensitivity to the $C_{\mathrm{RF}}$ and $k_{\perp}$ parameters is reported in reference [39]. Similarly to TOFOR and NE213, the best fit values of the $C_{\mathrm{RF}}$ and $k_{\perp}$ parameters were obtained and unconstrained statistical uncertainties for each parameter were estimated by a Monte-Carlo mapping of the likelihood function around the optimum. A confidence interval analysis showed that the parameters derived from the CVD were statistically compatible with those of the NE213 but not with TOFOR. Based on this further evidence, we have no reason to believe that there are instrumental inconsistencies in our data. The difference between parameters derived from observations along the vertical and the oblique line of sight must come from limitations of the one dimensional model (equation 2) in describing simultaneously observations along a dual sight line. This is discussed in the next section.

\section{Discussion}

5.1. Reconciling the apparently inconsistent measurements along vertical and oblique lines of sight

The analysis performed so far was based on a one dimensional model, which proved sufficient to describe neutron 


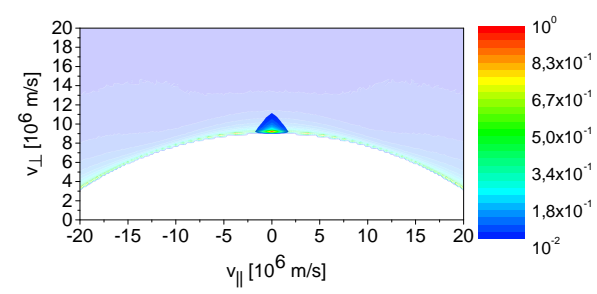

(a) $E_{n}=4 \mathrm{MeV}$

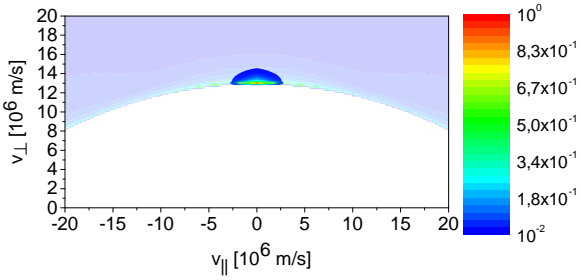

(c) $E_{n}=5 \mathrm{MeV}$

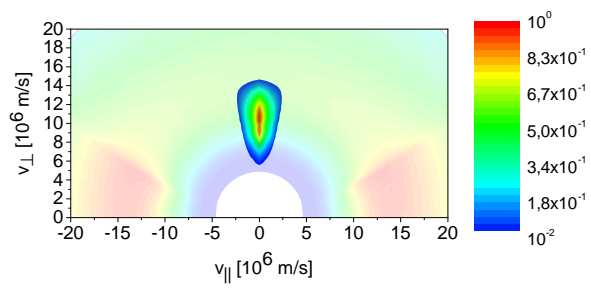

(b) $E_{\gamma}=2890 \mathrm{keV}$

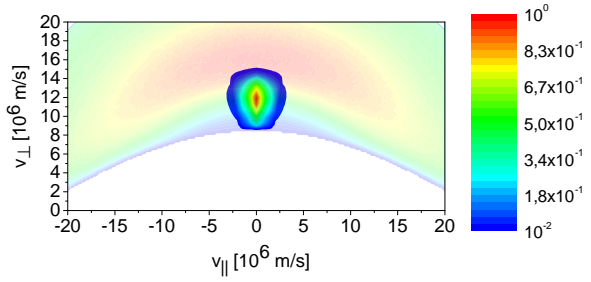

(d) $E_{\gamma}=2900 \mathrm{keV}$

Figure 10: Velocity space sensitivity of vertical neutron (left) and gamma-ray (right) measurements obtained by multiplication of the distribution function of discharge 86459 in the one dimensional model by the weight functions corresponding to the neutron/gamma-ray energy bin indicated below each figure. A pitch angle distributed as a Gaussian function with FWHM between 80 and 100 degrees was assumed. $\mathrm{x}$ and $\mathrm{y}$ axes show the parallel and perpendicular components of the deuteron velocity. The shaded colour regions show weight functions before multiplication by the energy distribution.

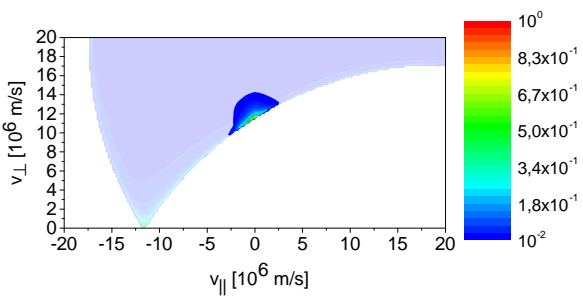

(a) $E_{n}=4 \mathrm{MeV}$

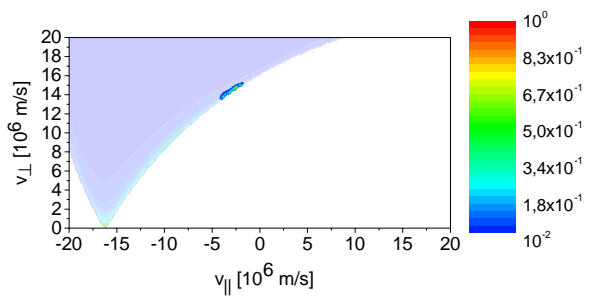

(b) $E_{n}=5 \mathrm{MeV}$

Figure 11: Velocity space sensitivity of oblique neutron spectroscopy measurements obtained by multiplication of the distribution function of discharge 86459 in the one dimensional model by the weight functions corresponding to the neutron energy bin indicated below each figure. A pitch angle distributed as a Gaussian function with FWHM between 80 and 100 degrees was assumed. x and y axes show the parallel and perpendicular components of the deuteron velocity. The shaded colour regions show weight functions before the multiplication by the energy distribution.

and gamma-ray spectroscopy data measured along a vertical line of sight in previous experiments [9, 11]. As anticipated in references [40, 41], however, neutron and gammaray spectroscopy measurements along an oblique line of sight may be sensitive to details of the pitch angle distribution away from 90 degrees, which are certainly not considered in the one dimensional model, where $v \approx v_{\perp}$ is assumed instead, i.e. all ions have a pitch angle of approximately 90 degrees. For a quantitative study on the sensitivity of a particular measurement technique to the fast ion velocity space, we can make use of the weight function formalism, which has recently been developed also for NES and GRS [40, 41] and can evaluate the velocity space regions that are observed when fast ions that undergo a fusion reaction lead to the emission of a neutron or gamma-ray in a particular energy bin of the spectrum. Results on the velocity space seen by neutron and gamma-ray measurements along the vertical line of sight of TOFOR and the HpGe are shown in figure 10; the velocity space associated to observations along the oblique line of sight of NE213/CVD is shown instead in figure 11. In order to obtain these figures, we have multiplied weight functions corresponding to the neutron/gamma-ray energy bins specified in the captions by the energy distribution of the one dimensional model 
for 86459 , when the pitch angle is distributed as a Gaussian function with FWHM between 80 and 100 degrees. Weight functions before the multiplication by the energy distribution are also shown as the shaded coloured regions in each figure. Fully white regions are thus not observable at all, as the the weight function is correspondingly null.

A comparison between the velocity space sensitivity of vertical neutron and gamma-ray measurements reveals that this is similar. There is almost a one to one correspondence between the energy of neutrons and that of the fast deuterons, which is slightly broadened for gamma-rays with energies falling in the tails of the $2868 \mathrm{keV}$ peak from the ${ }^{9} \mathrm{Be}(\mathrm{d}, \mathrm{n} \gamma){ }^{10} \mathrm{~B}$ reaction. This explains why, on one hand, the same one dimensional model that describes TOFOR data is also consistent with measurements from the HpGe. On the other hand, it also shows that TOFOR measurements are especially sensitive to variations of the energy distribution close to its cut-off, which in turn implies that the determination of $k_{\perp}$ and $C_{\mathrm{RF}}$ from TOFOR is very accurate. Concerning the pitch angle sensitivity of vertical measurements, we note that NES weight functions describing these are bounded by circular arcs of rather large radius that are axi-symmetric about the $v_{\perp}$ axis [40]. For narrow functions such as those typical for ICRH heating, the curvature of the weight functions becomes negligible and hence the sensitivity becomes almost independent of the $v_{\|}$coordinate. The same is true for weight functions describing two-step reaction GRS measurements [41]. This implies that the one dimensional model is sufficient to explain the data, as it was assumed empirically in earlier investigations $[9,11]$ and can be here established on theoretical basis by means of the weight functions.

As far as the oblique line of sight is concerned (see figure 11), the situation is different. Here there is still a narrow energy region in the deuteron velocity space that is contributed by neutrons with energy close to the cut-off, but the weight functions are lopsided towards negative parallel velocities. Therefore most signal is generated due to deuterons with anti-parallel velocity components. This shift is particularly pronounced at $E_{n}=5 \mathrm{MeV}$, where we even find that particles at a pitch angle of 90 degrees are not observable, suggesting that the one dimensional model is not suitable to describe data along this line of sight. Hence a two dimensional model providing information on both energy and the pitch-angle $\theta_{p}$ is required.

As countercheck of the different pitch angle sensitivity offered by measurements along the vertical and oblique lines of sight, we have repeated the TOFOR and NE213 data analysis by using a broader $\theta_{p}$ range for the deuteron distribution, between 70 and 110 degrees. $C_{\mathrm{RF}}, k_{\perp}$ and the corresponding distribution functions derived under this assumption are shown in figures 7 and 8 right and can be compared to the case when $\theta_{p}$ is between 80 and 100 degrees, as originally assumed. We note in particular that the $C_{R F}$ and $k_{\perp}$ values derived from TOFOR data are insensitive to the pitch angle interval, as expected. On the contrary, results from the NE213 depend on the width of the pitch angle. In this second analysis, we have arbitrarily chosen $\theta_{p}$ in the range 70 to 110 degrees as we could make the parameters derived from TOFOR and the NE213 agree by modifying the pitch angle in this way. This comes from the fact that, by broadening the pitch angle, more fast deuterons are allowed to move closer to parallel to the oblique sightline, which corresponds to a larger Doppler shift of neutrons emitted in this direction. A given value of $E^{*}$ therefore gives rise to neutrons of higher energy for the wider pitch angle range. The value of $E^{*}$ that matches the NE213 data is expected to be lower than for the narrow pitch angle range, which is indeed what is observed from figure 7.

There can be other sources of systematic uncertainties that may in principle affect the values of the parameters derived, besides the pitch angle sensitivity just discussed. For the NE213 spectrometer there can be an additional contribution arising from triton burn-up neutrons (TBN)[42]. These are neutrons produced in the DT reaction, where the tritons come from the $\mathrm{d}(\mathrm{d}, \mathrm{p}) \mathrm{t}$ reaction. TBN typically constitute about 1 percent of the total neutron emission from deuterium plasmas. This contribution was, however, found not essential to describe measurements and was not taken into account. For the TOFOR analysis, a possible source of systematic uncertainty comes from the need to include FLR effects in the spectrum calculations. As described in [34], this requires that an assumption is made about the spatial distribution of the fast deuterons. In this work, just like in [34], the spatial distribution is represented by a simple step function in the major radius coordinate $R$; all fast ions (with $E>150 \mathrm{keV}$ ) are assumed to be located within about $20 \mathrm{~cm}$ from the ICRH resonance position. The exact limits of the step function affects the shape of the calculated TOFOR spectrum. A sensitivity study showed that this can have an effect also on the estimated distribution, but on a lower level than the effect of the pitch angle range on the NE213 results (c.f. figure 7). In particular, the FLR effects affect only the low energy side of the neutron spectrum, which means that the location of $E^{*}$ (determined by the value of $k_{\perp}$ ) is largely unaffected.

These arguments support the hypothesis that it is the structure of the pitch angle around resonance that is manifested in the difference between measurements along the vertical and oblique line of sight. Therefore, for the first time at JET, we have the unprecedented opportunity to use neutron and gamma-ray spectroscopy data on a dual line of sight as a platform to validate in detail two dimensional energy/pitch-angle calculations of the distribution function of $\mathrm{MeV}$ range deuterons by ab initio models of radiofrequency heating at multiple harmonics in fusion plasmas. This is a unique capability of JET and is of particular relevance, for example, in view of predicting the performance of radio-frequency heating scenarios in present and next step high power tokamaks, such as second harmonic tritium acceleration in JET and ITER DT plasmas [43]. To this end, there is a widespread need to rely on models that have first been carefully validated against measurements 
of fast ions in the $\mathrm{MeV}$ range before codes are used to predict the performance of future experiments. The third harmonic heating scenario described in this paper is a natural platform for this benchmark in a deuterium plasma and a dedicated activity in this respect has just been initiated. In the next section we briefly present how the uniform analysis framework for NES and GRS developed in this work is extended for this validation activity, with examples taken from a first comparison with the ASCOT-RFOF codes $[44,45]$ for the purpose of illustration only. Here, we do not discuss the assumptions and details that are behind the radio frequency modelling, since this is left to forthcoming, dedicated publications [46].

Apart from benchmarking first principle models, our measurements also suggest that it may even be possible to infer 2D fast ion distribution functions directly from the measurements by tomographic inversion in velocity space [47] as was recently experimentally demonstrated for fast ion D alpha measurements of $\mathrm{E}<100 \mathrm{keV}$ NBI ions along three lines of sight [48]. Here we note that GRS and NES measurements, as any fast ion measurement, can be combined and inverted to make up for the fact that only two lines-ofsight are available [49]. The peculiarity of a tomographic inversion of NES and GRS measurements lies in their sensitivity to $\mathrm{MeV}$ range ions (unlike $<100 \mathrm{keV}$ ions for the fast ion D alpha diagnostics), with direct implications on $\alpha$ particle observations in a deuterium-tritium plasma.

\subsection{Validation of ab initio RF heating models}

The data presented in this paper were analysed on the basis of a one dimensional model of the deuteron energy distribution, which has been used as input to calculate the expected signal on the neutron and gamma-ray spectrometers used in the experiment within a uniform framework and for comparison with actual measurements. Besides having no resolution in the pitch angle, the $1 \mathrm{D}$ model is also not spatially resolved. Therefore, the energy distribution that is derived is to be seen as spatially averaged along each instrument line of sight, with a weight given by the probability of the fast ions to produce nuclear radiation and thus strongly biased towards the plasma core. The Monte Carlo framework adopted for neutron and gamma-ray calculations $[33,27]$ is, however, general and can be set up for integrating spatially resolved, arbitrary fuel ion distributions over a full 3-dimensional model of the viewing cone of a given instrument. This has been developed already for plasmas heated by NBI only [50, 51, 52], when the fast ion energy distribution is described by the NUBEAM code [53], and is here extended for coupling with RF heating models, such as PION [54], SPOT-RFOF [45] and ASCOTRFOF [44]. In all cases, samples taken from the energy distribution and specified by their $(\mathrm{R}, \mathrm{z})$ location, energy, pitch-angle and statistical weight are used to calculate the spectrum seen by a specific instrument, taking into account details of the line of sight and shadow effects resulting from the finite width of the fast ion Larmor radius [34, 11]. The synthetic neutron/gamma-ray energy spectra thus derived are finally convoluted with the known, instrument specific response function for comparison with measurement.

An example of such comparison is provided for the purpose of illustration in figure 12, where measured data taken with the full set of neutron and gamma-ray detectors considered in this paper is compared to simulated signals based on the energy distribution for discharge 86459 calculated with ASCOT-RFOF (figure 13). This code has provided the best match to data so far, but a comparison activity is ongoing also with results from different RF models.

In the case of ASCOT-RFOF, the fits show that there is generally a quite good agreement between simulations and measurements, which holds also at a quantitative level (normalised chi square $\approx 1$ ) for all instruments, but TOFOR (normalised chi square $\approx 2.3$ ). In this latter case, ASCOT-RFOF correctly predicts the high energy cut-off seen in the spectrum, but there is mismatch at intermediate energies between $\mathrm{E}_{n}=2.5$ and $\mathrm{E}_{n}=5 \mathrm{MeV}$ (corresponding to $\mathrm{t}_{\mathrm{TOF}}$ in the range 65 to $45 \mathrm{~ns}$ ), where the fit is systematically lower than data, which is also reflected in a mismatch at $E_{n}<2.5 \mathrm{MeV}$ ( $t_{\mathrm{TOF}}=65 \mathrm{~ns}$ ). Referring back to section 3 , this suggests that the $C_{\mathrm{RF}}$ coefficient that would describe the ASCOT-RFOF distribution in the one dimensional model is underestimated with respect to measurements. In other words, ASCOT-RFOF predicts that slightly fewer particle get accelerated by $\mathrm{RF}$ than measured, although that maximum energy at which deuterons are driven by radio-frequency heating is correctly determined. One important point to note is, however, that ASCOT-RFOF correctly determines the pitch angle structure of the distribution around resonance, as it well describes diamond, NE213 and TOFOR data close to the high energy cut-off of the spectrum. This also comes from the fact that ASCOT-RFOF provides a calculation of the spatial (as well as velocity space) fast ion distribution and, in particular, how the pitch angle structure depends on the spatial location. This information is necessary to simulate measured data as, for example, the vertical sight line mostly views particles at the tip of their banana orbit, while the oblique view mainly integrates signals from fast ions on the outer leg of their banana trajectory.

The spectrometers considered in this paper provide line integrated measurements and, as noted already, do not have spatial resolution. However, energy information of the spectrometers on a dual line of sight can be complemented by spatially resolved measurements of neutron emission by means of the JET neutron camera [55], which has been recently refurbished with a digital data acquisition $[56,57]$. The neutron camera can be used, for example, to validate predictions of the power deposition by the RF waves in the plasma volume. A comparison between measurements and ASCOT-RFOF modelling is shown in figure 12, where it is seen that there is a good qualitative agreement between simulations and measurements, although a full quantitative agreement is still missing, especially for channels 8 to 14 , corresponding to the edges 


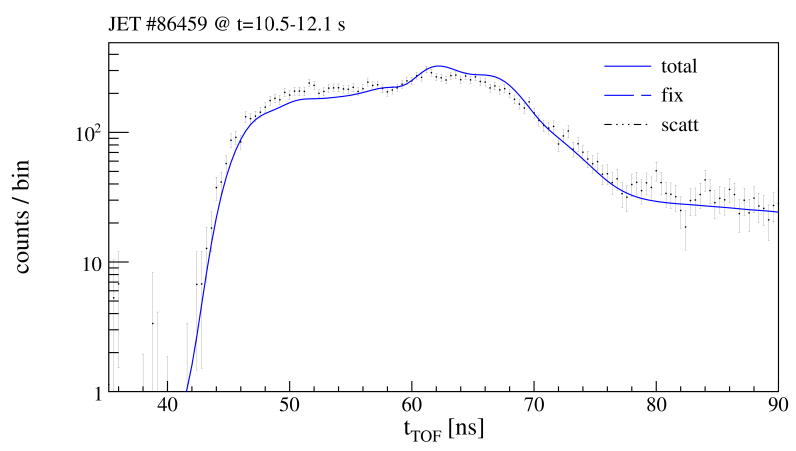

(a) TOFOR

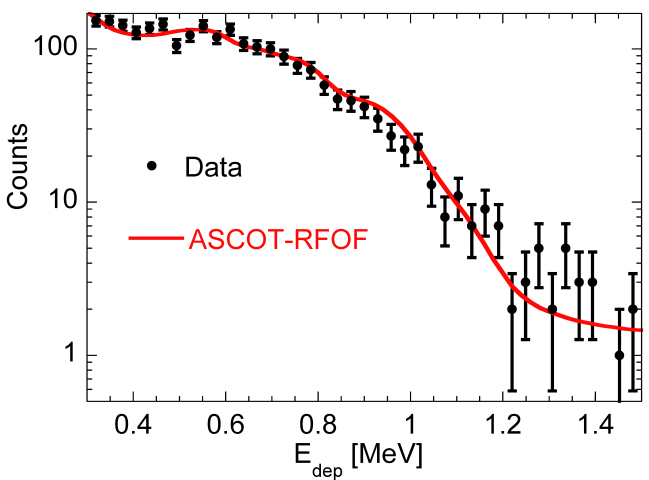

(c) Diamond

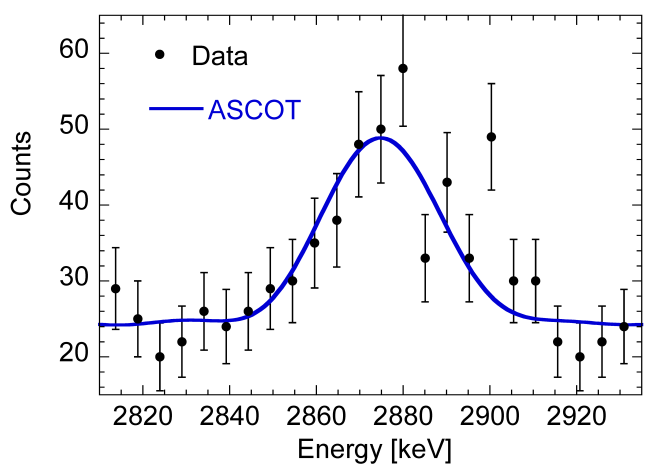

(e) $\mathrm{HpGe}-2868 \mathrm{keV}$

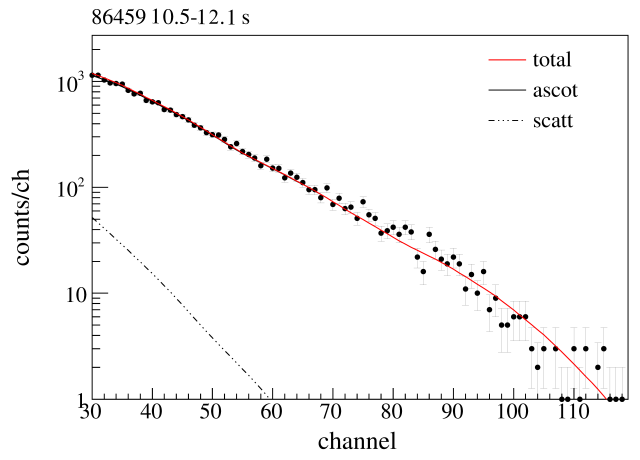

(b) NE213

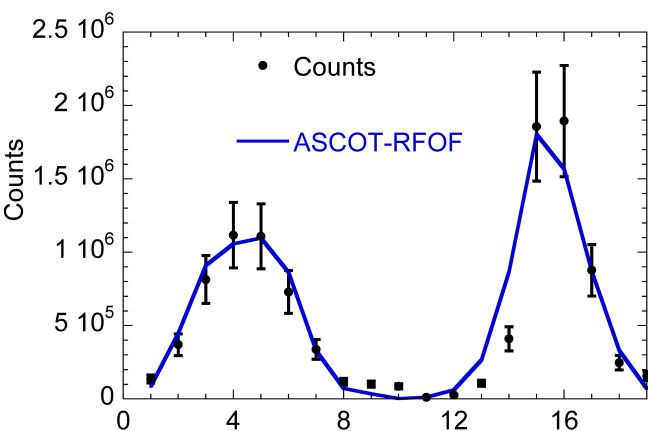

(d) Neutron Camera

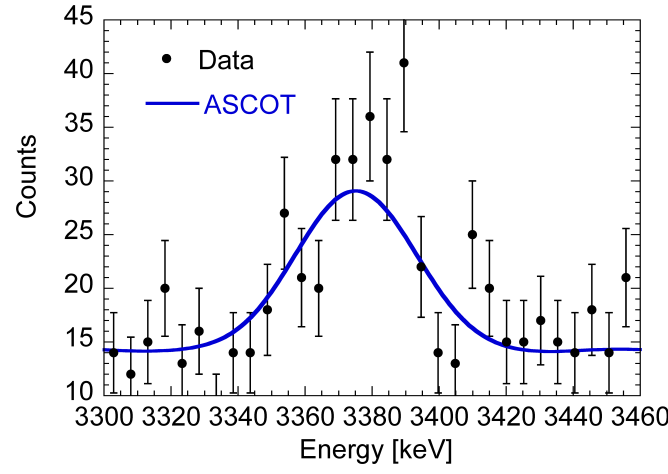

(f) $\mathrm{HpGe}-3367 \mathrm{keV}$

Figure 12: Comparison between data of different detectors (specified in the caption) and predictions based on the distribution function calculated by ASCOT-RFOF for 86459

of the neutron emission profile on the horizontal and vertical plane (see reference [55]). This may just reflect that a not fully correct magnetic equilibrium is being used in the ASCOT-RFOF simulations, for example due to the fact that the Shafranov shift of the flux surfaces is not precisely evaluated and is presently under investigation. In this preliminary comparison, we have also not considered absolute units (i.e. neutrons / second $/ \mathrm{cm}^{2}$ ) on the y axis of the neutron camera data, as the absolute calibration coefficients of this instrument, needed to convert from measured counts to neutron flux, are still to be evaluated.
Apart from these details, our first comparison between data and modelling already provides a convincing example of the advanced, unique and unprecedented possibilities offered by use of the combined set of JET neutron and gamma-ray diagnostics for detailed validation of first principle $\mathrm{RF}$ heating models of $\mathrm{MeV}$ range ions within the uniform analysis framework presented and in view of their exploitation to predict the performance of JET and ITER DT plasmas. The same framework, can also be used to assess models of the distribution function of any energetic ion in the $\mathrm{MeV}$ range, including its pitch angle 


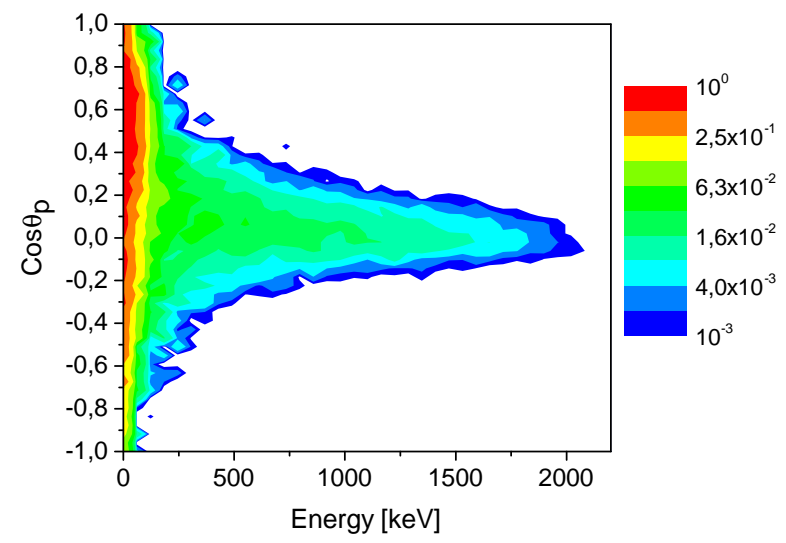

Figure 13: Steady state fast deuteron distribution function evaluated with ASCOT-RFOF for discharge 86459 and spatially integrated around the resonance layer between $\mathrm{R}=2.9$ and $\mathrm{R}=3.1 \mathrm{~m}$. The distribution is shown as a function of energy and the cosine of the pitch angle.

structure, with direct and highly interesting applications to studies of the $\alpha$ particle energy distribution in the next JET deuterium-tritium campaign.

Finally, we also point out that there is a certain degree of instrument redundancy as more than one detector is available on each of the two lines of sight. This is of importance to check for possible inconsistencies between different detectors and provides a high degree of reliability to the validation of RF models. However, we stress that even for identical lines of sight, the observed velocityspace and sensitivity within it is never identical for two different instruments, and so the measurements will also always give complementary information.

\section{Conclusions}

Experimental observations made in a JET experiment aimed at accelerating deuterons into the $\mathrm{MeV}$ range by a third harmonic ICRH experiment have been reported. The measurements were based on neutron and gamma-ray spectrometers observing the plasma, for the first time at JET, simultaneously along a vertical and oblique lines of sight. Data from the whole set of detectors were analysed to determine parameters of the fast ion energy distribution, within a uniform framework and a one dimensional model used to extract the energy cut-off in the deuteron phase space and the ICRH coupling constant from data. Differences between parameters derived from instruments viewing the plasma along the two lines of sight revealed that the combination of the two lines of sight makes the measurements sensitive to the two dimensional energy/pitchangle structure of the energy distribution, as confirmed by weight function calculations. This opens up to use of the collected data as a tool to validate ab initio models of ICRH at higher harmonics. A Monte Carlo framework for calculating synthetic diagnostic signals has been developed and a first comparison between data and predictions of the ASCOT-RFOF code has been illustrated.

The results presented in this paper are of relevance for the development of advanced diagnostic techniques for ions in the $\mathrm{MeV}$ range in high performance fusion plasmas, with applications to the detailed validation of radio-frequency heating models at multiple harmonics and for fusion product studies in forthcoming JET deuterium-tritium plasmas.

\section{Acknowledgment}

This work has been carried out within the framework of the EUROfusion Consortium and has received funding from the Euratom research and training programme 2014-2018 under grant agreement No 633053. The views and opinions expressed herein do not necessarily reflect those of the European Commission.

\section{References}

[1] Gorelenkov N, Pinches S and Toi K 2014 Nuclear Fusion 54125001

[2] Breizman B N and Sharapov S E 2011 Plasma Physics and Controlled Fusion $\mathbf{5 3} 054001$

[3] Garcia-Munoz M et al 2013 Nucl. Fusion 53123008

[4] Garcia-Munoz M et al 2013 Plasma Phys. Control. Fusion 55124014

[5] Mantsinen M J et al 2002 Phys. Rev. Lett. 88105002

[6] Eriksson L G et al 1998 Nuclear Fusion 38265

[7] Hellesen C et al 2010 Nucl. Fusion 50084006

[8] Stix T 1975 Nuclear Fusion 15737

[9] Hellesen C et al 2013 Nuclear Fusion 53113009 
[10] Gatu Johnson M et al 2010 Review of Scientific Instruments 81 10D336

[11] Nocente M et al 2012 Nuclear Fusion 52063009

[12] Shevelev A et al 2013 Nuclear Fusion 53123004

[13] Gatu Johnson M et al 2008 Nuclear Instruments and Methods in Physics Research Section A: Accelerators, Spectrometers, Detectors and Associated Equipment 591417 - 430

[14] Tardocchi M et al 2011 Phys. Rev. Lett. 107205002

[15] Tardocchi M, Nocente M and Gorini G 2013 Plasma Physics and Controlled Fusion 55074014

[16] Binda F et al 2014 Review of Scientific Instruments 85 11E123

[17] Cazzaniga C et al 2014 Rev. Sci. Instrum. 85043506

[18] Cazzaniga C et al 2014 Rev. Sci. Instrum. 85 11E101

[19] Andersson-Sunden E et al 2009 Nuclear Instruments and Methods in Physics Research Section A: Accelerators, Spectrometers, Detectors and Associated Equipment 610682 - 699

[20] Nocente M et al 2010 Rev. Sci. Instrum. 81 10D321

[21] Nocente M et al 2013 IEEE Trans. Nucl. Sci. 601408

[22] Cazzaniga C et al 2013 Rev. Sci. Instrum. 84123505

[23] Cazzaniga C et al 2015 Nucl. Instrum. Meth. A 778 20

[24] Kiptily V et al 2002 Nucl. Fusion 42999

[25] Romanelli F and JET-EFDA Contributors $2013 \mathrm{Nucl}$. Fusion $\mathbf{5 3} 104002$

[26] Proverbio I et al 2010 Rev. Sci. Instrum. 81 10D320

[27] Nocente M 2012 Neutron and gamma ray emission spectroscopy as fast ion diagnostics in fusion plasmas Ph.D. thesis Universitï œ di Milano-Bicocca URL http: //boa.unimib.it/handle/10281/28397

[28] Stix T 1992 Waves in plasmas (Chapter 17) (American Institute of Physics, New York.)

[29] Salmi A et al 2006 Plasma Physics and Controlled Fusion 48717

[30] Spitzer L 1962 The physics of fully ionized gases, 2nd edition (New York: Interscience)

[31] Stix T 1992 Waves in plasmas (Chapter 1) (American Institute of Physics, New York.)

[32] Mantsinen M et al 1999 Nucl. Fusion 39459

[33] Ballabio L, Gorini G and Källne J 1997 Physical Review E 55 3358-3368

[34] Eriksson J et al 2013 Plasma Physics and Controlled Fusion 55015008

[35] Nocente M et al 2011 Nucl. Fusion 51063011

[36] Nocente M et al 2013 Nucl. Fusion 53053010

[37] Gatu Johnson M et al 2010 Plasma Physics and Controlled Fusion $\mathbf{5 2} 085002$

[38] Binda F et al 'Neutron emission spectroscopy of third harmonic radio-frequency heated plasma with a NE213 scintillator at JET' Submitted to the Proceedings of the 1st EPS Conference on Plasma Diagnostics, Frascati, Italy, 2015

[39] Nocente M et al 2015 submitted to Rev. Sci. Instrum.

[40] Jacobsen A et al 2015 Nucl. Fusion 55053013
[41] Salewski M et al "Velocity-space observation regions of high-resolution two-step reaction gamma-ray spectroscopy" submitted to Nucl. Fusion

[42] Ballabio L et al 2000 Nucl. Fusion 4021

[43] ITER Physics Expert Group on Energetic Particles Heating and Current Drive and ITER Physics Basis Editors 1999 Nucl. Fusion 3921

[44] Hirvijoki E et al 2014 Computer Physics Communications 1851310

[45] Dumont R et al 2014 Europhysics Eonference Abstracts 38F P2.038

[46] Schneider M et al 'Modelling 3rd harmonic Ion Cyclotron acceleration of D beam for JET Fusion Product Studies experiments' In preparation for Nucl. Fusion

[47] Salewski M et al 2012 Nucl. Fusion 52103008

[48] Salewski M et al 2014 Nucl. Fusion 54023005

[49] Salewski M et al 2013 Nucl. Fusion 53063019

[50] Nocente M et al 2014 Nucl. Fusion 54104010

[51] Hellesen C et al 2010 Plasma Phys. Control. Fusion 52085013

[52] Chen Z et al 2013 Nucl. Fusion 53063023

[53] Pankin A et al 2004 Computer Physics Communications 159157

[54] Eriksson L G, Hellsten T and Willen U 1993 Nuclear Fusion 331037

[55] Jarvis O N 1994 Plasma Phys. Control. Fusion 36 209

[56] Giacomelli L et al 2014 Rev. Sci. Instrum. 85023505

[57] Giacomelli L et al 2015 Physics Procedia 62124 